\title{
Water Dynamics at the Solid-Liquid Interface to Unveil the Textural Features of Synthetic Nanosponges.
}

\author{
Paolo Lo Meo, ${ }^{* \dagger}$ Federico Mundo, ${ }^{\dagger}$ Samuele Terranova, ${ }^{\dagger}$ Pellegrino Conte, ${ }^{* \ddagger}$ Delia Chillura \\ Martino. $^{\dagger}$ \\ ${ }^{\dagger}$ Dept. of Biological, Chemical and Pharmaceutical Sciences and Technologies (STEBICEF), University of Palermo; \\ V.le delle Scienze ed. 17, 90128 Palermo, Italy. \\ ‡ Dept. of Agricoltural, Food and Forest Sciences (SAAF), University of Palermo; V.le delle Scienze ed. 4, 90128 \\ Palermo, Italy. \\ e-mail: paolo.lomeo@unipa.it; pellegrino.conte@unipa.it.
}

\section{Supporting Information}

\section{i) Short outline of FFC-NMR theory. ${ }^{1}$}

Fast field cycling (FFC) NMR relaxometry is the major technique to be applied in studying the behavior of a liquid system on the surface of a porous medium. In particular, as a solid material is added with liquid water, water molecules are subjected to two different types of motions. From the one hand, water molecules stay in the proximity of the solid surface for a short time during which they are subjected to a horizontal diffusion. From the other hand, molecules laying on the surface can leave it and reenters the bulk, while they are replaced by bulk water molecules. The distribution of water motional frequencies depends upon the homogeneity of the surface of the porous medium. Water confined in small-sized pores is more tightly constrained than that freely moving in larger spaces. The distributions of magnetic fields (DMF) due to the motional fluctuations are responsible for the dispersion of the longitudinal (or spin-lattice) relaxation times $\left(\mathrm{T}_{1}\right)$ occurring when each frequency in DMF matches the Larmor frequencies $\left(\omega_{\mathrm{L}}\right)$ of the observed nuclei. In the case of water containing systems, the observed nuclei are the protons in water molecules. Water near the surface can also interact with surficial paramagnetic systems. The resultant modulation of the local dipolar magnetic field generated by paramagnetism additionally contributes to spin-lattice relaxation. The direct relationship between the frequency of the water motion and the ${ }^{1} \mathrm{H}$ Larmor frequency is related to the water dynamics in porous media through the modulation of the intensity of the applied magnetic field. Fast field cycling NMR relaxometry is based on the fast change of the intensity of an applied magnetic field in order to monitor the variations of ${ }^{1} \mathrm{H}$ $\mathrm{T}_{1}$ values of a dynamic system.

Figure S1 shows the basic FFC NMR experimental design where the typical preparation-evolution-acquisition steps of the basic NMR experiment are replaced by polarization-relaxation-acquisition intervals. As outlined below, a pre-polarized (PP) and a non-polarized (NP) sequence can be recognized. During the first step of the PP sequence, a longitudinal magnetization is generated through the application of a polarization field ( $\mathrm{B}_{\mathrm{POL}}$ ) for a limited and fixed period of time (referred to as polarization time, $\mathrm{T}_{\mathrm{POL}}$ ). Afterwards, the magnetic field 
is switched to a new one (indicated as relaxation field, $B_{R L X}$ ), applied for a period $\tau$ during which the magnetization intensity relaxes to reach a new equilibrium condition. Finally, the application of a $1 \mathrm{H} 90^{\circ}$ pulse into an acquisition magnetic field ( $\mathrm{B}_{\mathrm{ACQ}}$ ) held for a fixed time makes the magnetization observable and the free induction decay (FID) acquirable. In the NP sequence, $B_{P O L}$ is null. The PP sequence is applied when the relaxation field becomes very low in intensity and enhancement of sensitivity is needed for FID achievement. The crossover field between the NP and PP sequences is approximately retrieved when the relaxation field intensity is half of that of the polarization field. The longitudinal relaxation time $\left(\mathrm{T}_{1}\right)$ values of the observed nuclei are obtained for every given $B_{R L X}$ through a progressive variation of the $\tau$ values.

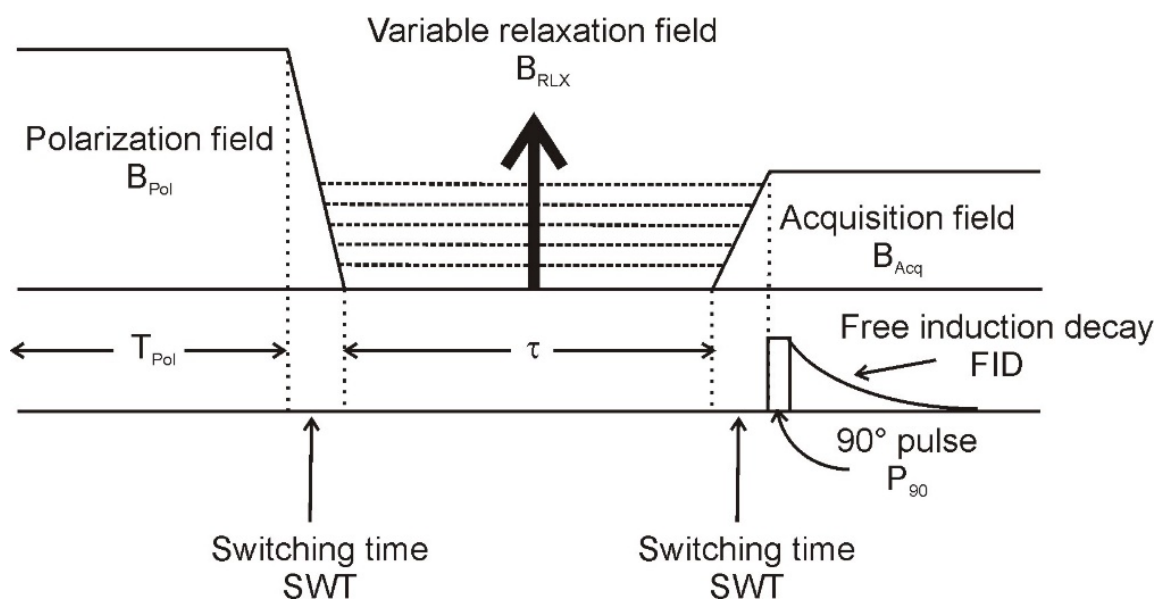

Figure S1. Scheme of the typical sequence used in FFC NMR relaxometry. The non-polarized sequence is obtained when $\mathrm{B}_{\mathrm{Pol}}$ is null. Conversely, as $\mathrm{B}_{\mathrm{Pol}}$ is non-null, the pre-polarized sequence is achieved.

The relationship between signal intensity and $\tau$ can be modeled as in equation (S1) for the PP sequence and as in equation (S2) for the NP sequence:

$M(\tau)=a+\sum_{i=1}^{N} b_{i} \exp \left\{-\frac{\tau}{T_{1 i}}\right\}$

$M(\tau)=a+\sum_{i=1}^{N} b_{i}\left(1-\exp \left\{-\frac{\tau}{T_{1 i}}\right\}\right)=\left(a+\sum_{i=1}^{N} b_{i}\right)-\sum_{i=1}^{N} b_{i} \exp \left\{-\frac{\tau}{T_{1 i}}\right\}$

In both equations (S1) and (S2), $M(\tau)$ is the magnetization intensity at the selected $\tau$ value; $a$ is the offset; $b_{i}$ is the magnetization intensity at the Boltzmann equilibrium of the i-th component of the molecular motion at each fixed $B_{R L X}$ intensity. Neither equations (S1) and (S2) can correctly represent the continuous distribution of $\mathrm{T}_{1}$ values when multiphase systems are investigated. When different components of the molecular dynamics in multiphase frames are described by longitudinal relaxation times with values very close to each other, a better representation of their $\mathrm{T}_{1}$ distributions can be obtained by applying an inverse Laplace transformation. It has the form reported in equation (S3) for the pre-polarized experiments, while it is as in equation (S4) when the non-polarized experiments are done. 
$M(\tau)=\int_{T_{1 \min }}^{T_{1 \max }} D\left(T_{1}\right) \exp \left[-\left(\frac{\tau}{T_{1}}\right)\right] d\left(T_{1}\right)+\sigma$

$M(\tau)=\int_{T_{1 \min }}^{T_{1 \max }} D\left(T_{1}\right)\left\{1-\exp \left[-\left(\frac{\tau}{T_{1}}\right)\right]\right\} d\left(T_{1}\right)+\sigma$

In equations (3) and (4), $T_{1 \min }$ and $T_{1 \max }$ are the longitudinal relaxation time limits within which all the $\mathrm{T}_{1}$ values fall. $D\left(T_{1}\right)$ is the distribution function which has to be determined by solving either equation (S3) or equation (S4); $\sigma$ is an unknown noise component. The latter makes impossible to find the exact distribution of relaxation times, thereby allowing infinite possible solutions for both the aforementioned equations. However, the most likely distribution of relaxation times can be obtained when some constraints, such as smoothness of the solution and variance of the experimental data, are accounted for. The algorithm used in the present study to retrieve the continuous $\mathrm{T}_{1}$ distributions the uniform penalty regularization, also referred to as UPEN.

The variations of the longitudinal relaxation rates $\left(R_{1}=1 / T_{1}\right)$ at each $B_{R L X}$ as described above, provide the nuclear magnetic resonance dispersion (NMRD) curves. These latter reflect the spectrum of re-orientational and diffusional molecular dynamics. They are described by Lorentzian functions with the form reported in equation (S5) through which information about correlation functions of the microscopic fluctuations can be achieved.

$R_{1}=\frac{1}{T_{1}}=\frac{\operatorname{Cons} t \times \tau_{C}}{1+\left(\omega_{L} \tau_{C}\right)^{2}}$

Here, Const is a constant parameter, $\omega_{\mathrm{L}}$ is the proton Larmor frequency (i.e., the $\mathrm{B}_{\mathrm{RLX}}$ value), and $\tau_{C}$ is the correlation time (i.e., the time taken for a molecule to rotate one radian or to move a distance of the order of its dimension). The longer the $\tau_{C}$ value, the slower the molecular motions are, thereby revealing restrictions in the motional freedom-degrees of spatially restrained molecular systems. Conversely, as a molecule encompasses faster motions due to higher degrees of freedom in larger spaces, shorter correlation time values are expected.

Equation (S5) is only valid when a non-stretched NMRD curve is obtained. When the NMR dispersion profiles are stretched as a consequence of the complexity of the re-orientational dynamics within the molecular system, the mathematical model developed by Halle and co-workers ${ }^{2}$ must be accounted for. It is also referred to as "model-free analysis" and is described as in equation (S6).

$R_{1}=\frac{1}{T_{1}}=\sum \frac{C_{i} \tau_{C i}}{1+\left(\omega_{L} \tau_{C}\right)^{2}}$

In equation (S6), $\omega_{L}$ is the proton Larmor frequency, $c_{i}$ is a fitting parameter, and $\tau_{C i}$ is the ith correlation time of the i-th component of the molecular system. The $c_{i}$ and $\tau_{C i}$ values are used as in equation (S7) to obtain an average correlation time of the whole system:

$\langle\tau\rangle=\frac{\sum_{i} c_{i} \tau_{C i}}{\sum_{i} c_{i}}$ 


\section{Bibliography}

1. Conte, P., Chapter 10 Environmental Applications of Fast Field-cycling NMR Relaxometry. In Field-cycling NMR Relaxometry: Instrumentation, Model Theories and Applications, The Royal Society of Chemistry: 2019; pp 229-254.

2. Halle, B.; Jóhannesson, H.; Venu, K., Model-Free Analysis of Stretched Relaxation Dispersions. J. Magn. Reson. 1998, 135, 1-13. 
ii) Table S1. $R_{1}$ vs $\omega_{L}$ data.

\begin{tabular}{|c|c|c|c|c|c|c|c|c|c|}
\hline \multicolumn{2}{|c|}{$\beta C D$} & \multicolumn{2}{|c|}{ NS1 dry } & \multicolumn{2}{|c|}{$\mathrm{NS} 1+\mathrm{H}_{2} \mathrm{O}$ fast } & \multicolumn{2}{|c|}{$\mathrm{NS} 1+\mathrm{H}_{2} \mathrm{O}$ slow } & \multicolumn{2}{|c|}{$\mathrm{NS} 1+\mathrm{D}_{2} \mathrm{O}$ fast } \\
\hline $\begin{array}{c}\omega_{\mathrm{L}} \\
(\mathrm{MHz})\end{array}$ & $\begin{array}{c}R_{1} \\
\left(\mathrm{~s}^{-1}\right) \\
\end{array}$ & $\begin{array}{c}\omega_{\mathrm{L}} \\
(\mathrm{MHz})\end{array}$ & $\begin{array}{c}R_{1} \\
\left(\mathrm{~s}^{-1}\right)\end{array}$ & $\begin{array}{c}\omega_{\mathrm{L}} \\
(\mathrm{MHz})\end{array}$ & $\begin{array}{c}R_{1} \\
\left(\mathrm{~s}^{-1}\right) \\
\end{array}$ & $\begin{array}{c}\omega_{\mathrm{L}} \\
(\mathrm{MHz})\end{array}$ & $\begin{array}{c}R_{1} \\
\left(\mathrm{~s}^{-1}\right) \\
\end{array}$ & $\begin{array}{c}\omega_{\mathrm{L}} \\
(\mathrm{MHz})\end{array}$ & $\begin{array}{c}R_{1} \\
\left(\mathrm{~s}^{-1}\right) \\
\end{array}$ \\
\hline 0.3 & $175 \pm 17$ & 0.3 & $320 \pm 3$ & 0.1 & $42 \pm 5$ & 0.1 & $4.33 \pm 0.09$ & 0.4 & $250 \pm 25$ \\
\hline 0.7 & $102 \pm 6$ & 0.7 & $192 \pm 2$ & 0.3 & $40 \pm 5$ & 0.3 & $3.78 \pm 0.10$ & 0.6 & $256 \pm 26$ \\
\hline 1.0 & $66 \pm 3$ & 1.0 & $142.8 \pm 1.4$ & 0.7 & $38 \pm 3$ & 0.7 & $3.17 \pm 0.05$ & 0.8 & $189 \pm 21$ \\
\hline 1.3 & $56 \pm 3$ & 1.3 & $120.9 \pm 0.9$ & 1.0 & $40 \pm 3$ & 1.0 & $3.04 \pm 0.05$ & 1.3 & $116 \pm 9$ \\
\hline 1.5 & $47 \pm 3$ & 1.5 & $110.2 \pm 1.0$ & 2.0 & $34 \pm 2$ & 2.0 & $2.70 \pm 0.04$ & 1.5 & $98 \pm 9$ \\
\hline 1.7 & $43 \pm 2$ & 1.7 & $98.3 \pm 0.7$ & 2.1 & $36 \pm 3$ & 2.1 & $2.75 \pm 0.05$ & 1.8 & $93 \pm 7$ \\
\hline 2.0 & $33.5 \pm 1.3$ & 2.0 & $88.3 \pm 0.9$ & 2.2 & $34 \pm 2$ & 2.2 & $2.73 \pm 0.05$ & 2 & $83 \pm 7$ \\
\hline 2.5 & $34.0 \pm 1.1$ & 2.1 & $88.2 \pm 0.5$ & 2.3 & $35 \pm 2$ & 2.3 & $2.70 \pm 0.04$ & 2.1 & $83 \pm 14$ \\
\hline 3.0 & $28.0 \pm 1.0$ & 2.2 & $87.8 \pm 0.7$ & 2.4 & $36 \pm 3$ & 2.4 & $2.70 \pm 0.04$ & 2.2 & $77 \pm 6$ \\
\hline 4.0 & $17.7 \pm 0.7$ & 2.3 & $88.1 \pm 0.7$ & 2.5 & $33 \pm 2$ & 2.45 & $2.72 \pm 0.04$ & 2.3 & $100 \pm 10$ \\
\hline 4.5 & $16.4 \pm 0.5$ & 2.35 & $93.9 \pm 0.9$ & 2.6 & $37 \pm 3$ & 2.5 & $2.67 \pm 0.04$ & 2.4 & $77 \pm 12$ \\
\hline 5.0 & $16.0 \pm 0.5$ & 2.4 & $97.1 \pm 0.9$ & 2.7 & $33 \pm 2$ & 2.6 & $2.68 \pm 0.04$ & 2.45 & $62 \pm 27$ \\
\hline 6.0 & $12 \pm 2$ & 2.45 & $99.3 \pm 0.8$ & 2.8 & $32 \pm 2$ & 2.7 & $2.66 \pm 0.04$ & 2.5 & $77 \pm 12$ \\
\hline 8.0 & $8.8 \pm 0.9$ & 2.5 & $100.9 \pm 0.8$ & 2.9 & $37 \pm 3$ & 2.8 & $2.62 \pm 0.04$ & 2.6 & $77 \pm 6$ \\
\hline 9.0 & $8.4 \pm 0.5$ & 2.55 & $101.0 \pm 0.6$ & 3.0 & $35 \pm 2$ & 2.9 & $2.64 \pm 0.04$ & 2.7 & $91 \pm 50$ \\
\hline 10.0 & $8.1 \pm 0.6$ & 2.6 & $97.5 \pm 0.8$ & 3.05 & $37 \pm 3$ & 3.0 & $2.60 \pm 0.04$ & 2.8 & $63 \pm 4$ \\
\hline 12.0 & $6.9 \pm 0.4$ & 2.7 & $97.8 \pm 1.0$ & 3.1 & $37 \pm 3$ & 3.05 & $2.65 \pm 0.04$ & 2.9 & $71 \pm 20$ \\
\hline 15.0 & $5.7 \pm 0.3$ & 2.8 & $92.8 \pm 0.9$ & 3.15 & $32 \pm 2$ & 3.1 & $2.63 \pm 0.04$ & 3.0 & $71 \pm 10$ \\
\hline 17.0 & $4.9 \pm 0.2$ & 2.9 & $86.3 \pm 0.7$ & 3.2 & $32 \pm 2$ & 3.15 & $2.61 \pm 0.03$ & 3.05 & $67 \pm 13$ \\
\hline 20.0 & $4.6 \pm 0.1$ & 3.0 & $91.4 \pm 0.8$ & 3.3 & $33 \pm 2$ & 3.2 & $2.56 \pm 0.04$ & 3.1 & $53 \pm 6$ \\
\hline 25.0 & $4.0 \pm 0.1$ & 3.05 & $98.7 \pm 0.7$ & 3.4 & $31 \pm 2$ & 3.3 & $2.55 \pm 0.03$ & 3.15 & $71 \pm 15$ \\
\hline \multirow[t]{23}{*}{30.0} & $3.6 \pm 0.1$ & 3.1 & $105.3 \pm 1.0$ & 3.5 & $29 \pm 2$ & 3.4 & $2.51 \pm 0.04$ & 3.2 & $56 \pm 6$ \\
\hline & & 3.15 & $107.1 \pm 1.0$ & 4.0 & $25.6 \pm 1.3$ & 3.5 & $2.48 \pm 0.04$ & 3.3 & $53 \pm 6$ \\
\hline & & 3.2 & $100.8 \pm 0.9$ & 5.0 & $22.2 \pm 1.5$ & 4.0 & $2.38 \pm 0.03$ & 3.4 & $56 \pm 6$ \\
\hline & & 3.3 & $91.2 \pm 1.0$ & 6.0 & $20.8 \pm 1.3$ & 5.0 & $2.26 \pm 0.03$ & 3.45 & $53 \pm 3$ \\
\hline & & 3.4 & $73.7 \pm 0.5$ & 7.0 & $18.5 \pm 1.0$ & 6.0 & $2.18 \pm 0.04$ & 3.47 & $43 \pm 6$ \\
\hline & & 3.5 & $59.9 \pm 0.7$ & 8.0 & $18.5 \pm 1.0$ & 7.0 & $2.07 \pm 0.03$ & 3.5 & $48 \pm 2$ \\
\hline & & 4.0 & $50.5 \pm 0.4$ & 9.0 & $15.9 \pm 1.0$ & 8.0 & $2.05 \pm 0.03$ & 3.8 & $42 \pm 2$ \\
\hline & & 5.0 & $41.7 \pm 0.6$ & 10.0 & $15.6 \pm 0.7$ & 9.0 & $1.94 \pm 0.03$ & 4.0 & $42 \pm 3$ \\
\hline & & 6.0 & $34.8 \pm 0.5$ & 15.0 & $12.5 \pm 0.5$ & 10.0 & $1.93 \pm 0.02$ & 5.0 & $32 \pm 2$ \\
\hline & & 7.0 & $31.5 \pm 0.5$ & 20.0 & $10.3 \pm 0.2$ & 15.0 & $1.74 \pm 0.02$ & 6.0 & $29 \pm 2$ \\
\hline & & 8.0 & $26.8 \pm 0.4$ & 25.0 & $9.0 \pm 0.2$ & 20.0 & $1.62 \pm 0.01$ & 7.0 & $26 \pm 3$ \\
\hline & & 9.0 & $24.3 \pm 0.5$ & 30.0 & $7.8 \pm 0.2$ & 25.0 & $1.53 \pm 0.01$ & 8.0 & $31 \pm 4$ \\
\hline & & 10.0 & $22.1 \pm 0.2$ & 35.0 & $7.1 \pm 0.2$ & 30.0 & $1.44 \pm 0.01$ & 9.0 & $24 \pm 4$ \\
\hline & & 11.0 & $20.0 \pm 0.3$ & & & 35.0 & $1.38 \pm 0.01$ & 10.0 & $18 \pm 2$ \\
\hline & & 12.0 & $18.8 \pm 0.3$ & & & & & 12.0 & $16.7 \pm 1.4$ \\
\hline & & 13.0 & $17.1 \pm 0.2$ & & & & & 15.0 & $12.2 \pm 0.7$ \\
\hline & & 14.0 & $16.3 \pm 0.2$ & & & & & 20.0 & $9.4 \pm 0.7$ \\
\hline & & 15.0 & $15.1 \pm 0.2$ & & & & & 25.0 & $7.1 \pm 0.5$ \\
\hline & & 17.0 & $13.5 \pm 0.1$ & & & & & 30.0 & $5.3 \pm 0.3$ \\
\hline & & 20.0 & $12.0 \pm 0.1$ & & & & & 35.0 & $5.0 \pm 0.2$ \\
\hline & & 25.0 & $9.7 \pm 0.1$ & & & & & & \\
\hline & & 30.0 & $8.1 \pm 0.1$ & & & & & & \\
\hline & & 35.0 & $7.1 \pm 0.1$ & & & & & & \\
\hline
\end{tabular}


Table S1 (follows).

\begin{tabular}{|c|c|c|c|c|c|c|c|}
\hline \multicolumn{2}{|c|}{ NS2 dry } & \multicolumn{2}{|c|}{$\mathrm{NS} 2+\mathrm{H}_{2} \mathrm{O}$ fast } & \multicolumn{2}{|c|}{$\mathrm{NS} 2+\mathrm{H}_{2} \mathrm{O}$ slow } & \multicolumn{2}{|l|}{ NS3 dry } \\
\hline $\begin{array}{c}\omega_{\mathrm{L}} \\
(\mathrm{MHz})\end{array}$ & $\begin{array}{c}R_{1} \\
\left(\mathrm{~s}^{-1}\right) \\
\end{array}$ & $\begin{array}{c}\omega \mathrm{L} \\
(\mathrm{MHz})\end{array}$ & $\begin{array}{c}R_{1} \\
\left(\mathrm{~s}^{-1}\right)\end{array}$ & $\begin{array}{c}\omega \mathrm{L} \\
(\mathrm{MHz})\end{array}$ & $\begin{array}{c}R_{1} \\
\left(\mathrm{~s}^{-1}\right)\end{array}$ & $\omega_{\mathrm{L}}(\mathrm{MHz})$ & $\begin{array}{c}R_{1} \\
\left(\mathrm{~s}^{-1}\right)\end{array}$ \\
\hline 0.3 & $387 \pm 11$ & 0.1 & $38 \pm 2$ & 0.1 & $1.66 \pm 0.05$ & 0.7 & $218 \pm 16$ \\
\hline 0.7 & $240 \pm 4$ & 0.3 & $38 \pm 2$ & 0.3 & $1.64 \pm 0.03$ & 1.0 & $181 \pm 33$ \\
\hline 1.0 & $167 \pm 3$ & 0.7 & $32 \pm 2$ & 0.7 & $1.59 \pm 0.03$ & 1.3 & $134 \pm 4$ \\
\hline 1.3 & $151 \pm 3$ & 1.0 & $38 \pm 3$ & 1.0 & $1.56 \pm 0.02$ & 1.5 & $115 \pm 4$ \\
\hline 1.5 & $126 \pm 3$ & 1.3 & $38 \pm 3$ & 1.3 & $1.54 \pm 0.02$ & 1.7 & $100 \pm 3$ \\
\hline 1.7 & $121 \pm 2$ & 1.5 & $38 \pm 3$ & 1.5 & $1.52 \pm 0.02$ & 2.0 & $81 \pm 2$ \\
\hline 1.85 & $112 \pm 2$ & 1.7 & $36 \pm 3$ & 1.7 & $1.47 \pm 0.02$ & 2.1 & $85 \pm 2$ \\
\hline 2.0 & $104 \pm 2$ & 1.85 & $34 \pm 2$ & 1.85 & $1.47 \pm 0.02$ & 2.2 & $78 \pm 2$ \\
\hline 2.1 & $102 \pm 2$ & 2.0 & $34 \pm 2$ & 2 & $1.49 \pm 0.02$ & 2.3 & $77 \pm 2$ \\
\hline 2.2 & $99 \pm 2$ & 2.1 & $37 \pm 3$ & 2.1 & $1.49 \pm 0.02$ & 2.35 & $79 \pm 2$ \\
\hline 2.3 & $103 \pm 2$ & 2.3 & $36 \pm 3$ & 2.3 & $1.47 \pm 0.02$ & 2.4 & $74 \pm 2$ \\
\hline 2.35 & $107 \pm 2$ & 2.35 & $38 \pm 3$ & 2.35 & $1.45 \pm 0.02$ & 2.45 & $85 \pm 2$ \\
\hline 2.45 & $118 \pm 2$ & 2.4 & $37 \pm 3$ & 2.4 & $1.45 \pm 0.02$ & 2.5 & $75 \pm 2$ \\
\hline 2.5 & $114 \pm 2$ & 2.45 & $37 \pm 3$ & 2.45 & $1.45 \pm 0.02$ & 2.55 & $76 \pm 2$ \\
\hline 2.55 & $118 \pm 2$ & 2.5 & $37 \pm 3$ & 2.5 & $1.45 \pm 0.02$ & 2.6 & $80 \pm 3$ \\
\hline 2.6 & $116 \pm 2$ & 2.55 & $37 \pm 3$ & 2.55 & $1.45 \pm 0.02$ & 2.7 & $74 \pm 2$ \\
\hline 2.7 & $110 \pm 2$ & 2.6 & $37 \pm 3$ & 2.6 & $1.45 \pm 0.02$ & 2.8 & $75 \pm 2$ \\
\hline 2.8 & $97.3 \pm 1.3$ & 2.7 & $36 \pm 3$ & 2.7 & $1.43 \pm 0.02$ & 2.9 & $69 \pm 2$ \\
\hline 2.9 & $99 \pm 2$ & 2.8 & $34 \pm 2$ & 2.8 & $1.43 \pm 0.02$ & 3.0 & $67 \pm 2$ \\
\hline 2.95 & $96 \pm 2$ & 2.9 & $36 \pm 3$ & 2.9 & $1.43 \pm 0.02$ & 3.05 & $61 \pm 2$ \\
\hline 3.0 & $106 \pm 2$ & 2.95 & $36 \pm 3$ & 2.95 & $1.43 \pm 0.02$ & 3.1 & $69 \pm 3$ \\
\hline 3.1 & $119 \pm 2$ & 3.0 & $37 \pm 3$ & 3 & $1.45 \pm 0.02$ & 3.15 & $70 \pm 2$ \\
\hline 3.15 & $125 \pm 3$ & 3.1 & $37 \pm 3$ & 3.1 & $1.43 \pm 0.02$ & 3.2 & $65 \pm 2$ \\
\hline 3.2 & $114 \pm 2$ & 3.15 & $36 \pm 3$ & 3.15 & $1.43 \pm 0.02$ & 3.3 & $65 \pm 2$ \\
\hline 3.3 & $98 \pm 2$ & 3.2 & $34 \pm 2$ & 3.2 & $1.43 \pm 0.02$ & 3.4 & $60 \pm 2$ \\
\hline 3.4 & $79 \pm 2$ & 3.3 & $33 \pm 2$ & 3.3 & $1.41 \pm 0.02$ & 3.5 & $52 \pm 2$ \\
\hline 3.5 & $67.6 \pm 1.3$ & 3.4 & $31 \pm 2$ & 3.4 & $1.39 \pm 0.02$ & 4.0 & $45.0 \pm 1.4$ \\
\hline 3.7 & $62.0 \pm 1.0$ & 3.5 & $30 \pm 2$ & 3.5 & $1.33 \pm 0.02$ & 5.0 & $35.4 \pm 1.1$ \\
\hline 4.0 & $57.8 \pm 1.1$ & 3.7 & $30 \pm 2$ & 3.7 & $1.37 \pm 0.02$ & 6.0 & $25.8 \pm 1.0$ \\
\hline 5.0 & $45.9 \pm 1.0$ & 4.0 & $28 \pm 2$ & 4.0 & $1.35 \pm 0.06$ & 7.0 & $26.1 \pm 0.8$ \\
\hline 6.0 & $37.0 \pm 0.8$ & 5.0 & $25.0 \pm 1.3$ & 5.0 & $1.32 \pm 0.02$ & 8.0 & $19.5 \pm 0.7$ \\
\hline 7.0 & $34.5 \pm 0.7$ & 6.0 & $23.3 \pm 1.1$ & 6.0 & $1.27 \pm 0.02$ & 9.0 & $18.2 \pm 0.7$ \\
\hline 8.0 & $28.4 \pm 0.8$ & 7.0 & $22.7 \pm 1.0$ & 7.0 & $1.23 \pm 0.02$ & 10.0 & $13.8 \pm 0.6$ \\
\hline 10.0 & $22.7 \pm 0.7$ & 8.0 & $20.8 \pm 0.9$ & 8.0 & $1.22 \pm 0.01$ & 11.0 & $12.7 \pm 0.7$ \\
\hline 11.0 & $23.4 \pm 1.0$ & 10.0 & $18.2 \pm 0.7$ & 10.0 & $1.18 \pm 0.01$ & 12.0 & $10.6 \pm 0.6$ \\
\hline 12.0 & $18.9 \pm 0.8$ & 11.0 & $17.2 \pm 0.6$ & 11.0 & $1.16 \pm 0.01$ & 13.0 & $10.4 \pm 0.3$ \\
\hline 13.0 & $17.4 \pm 0.4$ & 12.0 & $15.9 \pm 0.5$ & 12.0 & $1.14 \pm 0.01$ & 14.0 & $9.7 \pm 0.4$ \\
\hline 14.0 & $15.3 \pm 0.2$ & 13.0 & $15.6 \pm 0.5$ & 13.0 & $1.13 \pm 0.01$ & 15.0 & $9.2 \pm 0.3$ \\
\hline 15.0 & $14.2 \pm 0.2$ & 14.0 & $14.9 \pm 0.4$ & 14.0 & $1.11 \pm 0.01$ & 17.0 & $8.4 \pm 0.3$ \\
\hline 17.0 & $13.3 \pm 0.2$ & 15.0 & $13.9 \pm 0.4$ & 15.0 & $1.09 \pm 0.01$ & 20.0 & $7.3 \pm 0.2$ \\
\hline 20.0 & $11.2 \pm 0.1$ & 17.0 & $13.2 \pm 0.5$ & 17.0 & $1.08 \pm 0.01$ & 25.0 & $5.81 \pm 0.12$ \\
\hline 25.0 & $9.6 \pm 0.1$ & 20.0 & $12.2 \pm 0.4$ & 20.0 & $1.04 \pm 0.01$ & 30.0 & $4.98 \pm 0.10$ \\
\hline 30.0 & $7.94 \pm 0.07$ & 25.0 & $10.8 \pm 0.7$ & 25.0 & $1.01 \pm 0.03$ & 35.0 & $4.26 \pm 0.06$ \\
\hline 35.0 & $6.90 \pm 0.05$ & 30.0 & $10.3 \pm 0.9$ & 30.0 & $0.97 \pm 0.03$ & & \\
\hline & & 35.0 & $7.8 \pm 0.4$ & 35.0 & $0.93 \pm 0.03$ & & \\
\hline
\end{tabular}


iii) Table S2. Halle's regression parameters $R_{1}$ vs $\omega_{\mathrm{L}}$ curves.

$$
R_{1}=\sum_{i} \frac{c_{i} \tau_{i}}{1+\left(\omega_{L} \tau_{i}\right)^{2}}+\sum_{j_{(d i p s)}}\left[\frac{2}{\pi} \cdot \frac{c_{j} s_{j}}{4\left(\omega_{L}-\omega_{j}\right)^{2}+s_{j}^{2}}\right]
$$

\begin{tabular}{|c|c|c|c|c|c|}
\hline sample & $c_{\mathrm{i}}$ & $\tau_{\mathrm{i}}(\mu \mathrm{s})$ & $c_{\mathrm{j}}$ & $s_{\mathrm{j}}(\mathrm{MHz})$ & $\omega_{\mathrm{j}}(\mathrm{MHz})$ \\
\hline \multirow[t]{3}{*}{$\beta C D$} & $209 \pm 12$ & $0.021 \pm 0.003$ & - & - & - \\
\hline & $101 \pm 10$ & $0.31 \pm 0.05$ & - & - & - \\
\hline & $94 \pm 13$ & $2.2 \pm 0.6$ & - & - & - \\
\hline \multirow[t]{3}{*}{ NS1 dry } & $414 \pm 8$ & $0.027 \pm 0.002$ & $22 \pm 2$ & $0.33 \pm 0.03$ & $3.16 \pm 0.01$ \\
\hline & $252 \pm 13$ & $0.20 \pm 0.02$ & $27 \pm 4$ & $0.57 \pm 0.07$ & $2.57 \pm 0.02$ \\
\hline & $192 \pm 10$ & $1.60 \pm 0.11$ & & & \\
\hline $\mathrm{NS} 1+\mathrm{H}_{2} \mathrm{O}$ & $466 \pm 9$ & $0.0240 \pm 0.0014$ & $3.7 \pm 1.4$ & $0.5 \pm 0.2$ & $3.03 \pm 0.05$ \\
\hline “fast” & $125 \pm 7$ & $0.233 \pm 0.014$ & & & \\
\hline $\mathrm{NS} 1+\mathrm{H}_{2} \mathrm{O}$ & $0.53 \pm 0.06$ & $2.9 \pm 0.3$ & & & \\
\hline \multirow[t]{2}{*}{ “slow” } & $5.9 \pm 0.5$ & $0.202 \pm 0.015$ & & & \\
\hline & $114 \pm 7$ & $0.0149 \pm 0.0012$ & & & \\
\hline $\mathrm{NS} 1+\mathrm{D}_{2} \mathrm{O}$ & $375 \pm 12$ & $0.060 \pm 0.004$ & $57 \pm 28$ & $1.6 \pm 0.4$ & $2.5 \pm 0.2$ \\
\hline “fast” & $253 \pm 21$ & $1.2 \pm 0.2$ & & & \\
\hline \multirow[t]{3}{*}{ NS2 dry } & $405 \pm 12$ & $0.021 \pm 0.003$ & $24 \pm 2$ & $0.30 \pm 0.03$ & $3.15 \pm 0.01$ \\
\hline & $305 \pm 14$ & $0.19 \pm 0.02$ & $27 \pm 4$ & $0.48 \pm 0.07$ & $2.56 \pm 0.02$ \\
\hline & $246 \pm 14$ & $1.48 \pm 0.13$ & & & \\
\hline $\mathrm{NS} 2+\mathrm{H}_{2} \mathrm{O}$ & $526 \pm 16$ & $0.0250 \pm 0.0016$ & $2.3 \pm 0.8$ & $0.27 \pm 0.12$ & $3.07 \pm 0.03$ \\
\hline “fast” & $132 \pm 8$ & $0.188 \pm 0.011$ & $3 \pm 2$ & $0.5 \pm 0.3$ & $2.49 \pm 0.07$ \\
\hline $\mathrm{NS} 2+\mathrm{H}_{2} \mathrm{O}$ & $0.12 \pm 0.06$ & $1.2 \pm 0.5$ & & & \\
\hline \multirow[t]{2}{*}{ “slow” } & $2.2 \pm 0.2$ & $0.19 \pm 0.02$ & & & \\
\hline & $79 \pm 7$ & $0.0139 \pm 0.0014$ & & & \\
\hline \multirow[t]{3}{*}{ NS3 dry } & $257 \pm 26$ & $0.018 \pm 0.005$ & $11 \pm 6$ & $0.6 \pm 0.3$ & $2.68 \pm 0.06$ \\
\hline & $233 \pm 33$ & $0.22 \pm 0.05$ & $5 \pm 3$ & $0.3 \pm 0.2$ & $3.26 \pm 0.04$ \\
\hline & $244 \pm 39$ & $1.5 \pm 0.6$ & & & \\
\hline
\end{tabular}


iv) Normalized Inverse-Laplace (UPEN) $T_{1}$ distribution curves.

Figure S1. $T_{1}$ distribution curves at $35 \mathrm{MHz}$.

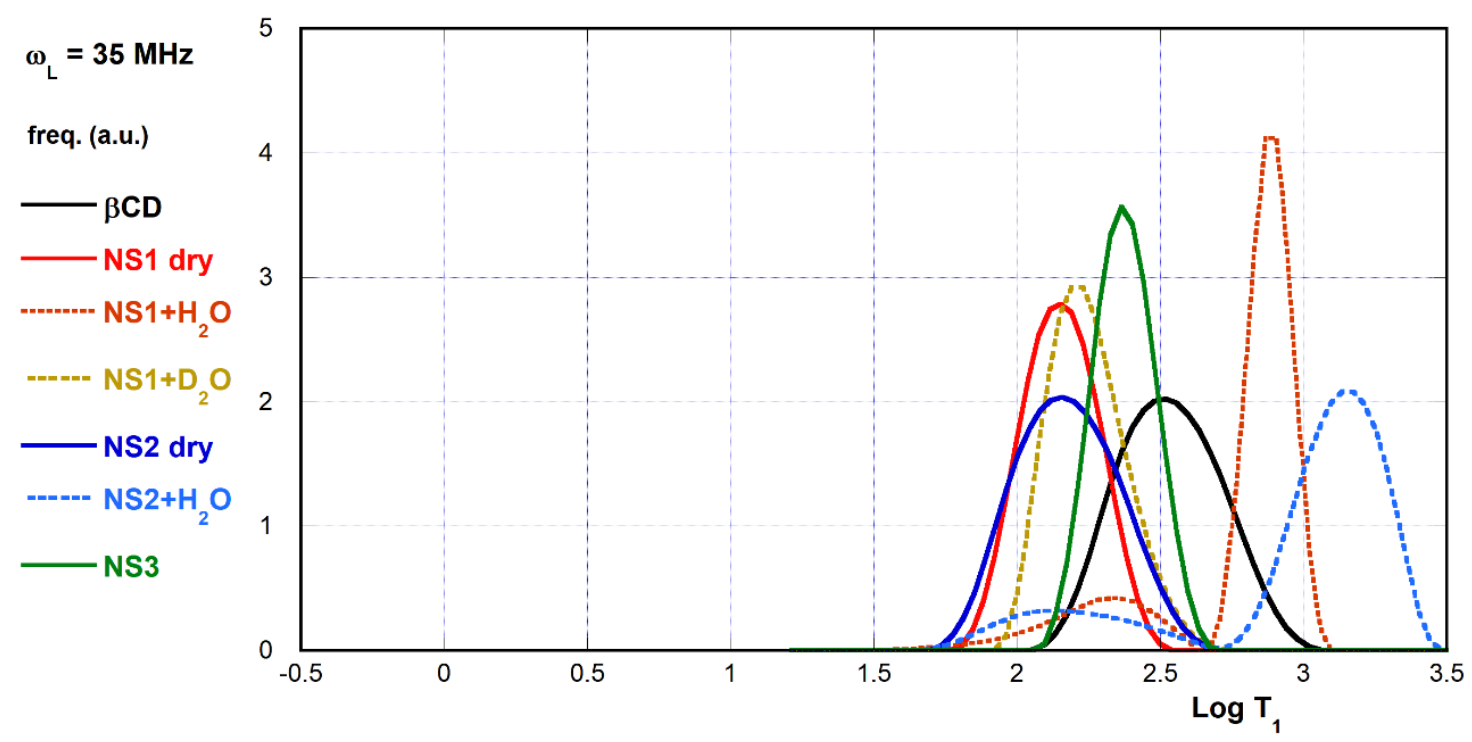

Figure S2. $T_{1}$ distribution curves at $10 \mathrm{MHz}$.

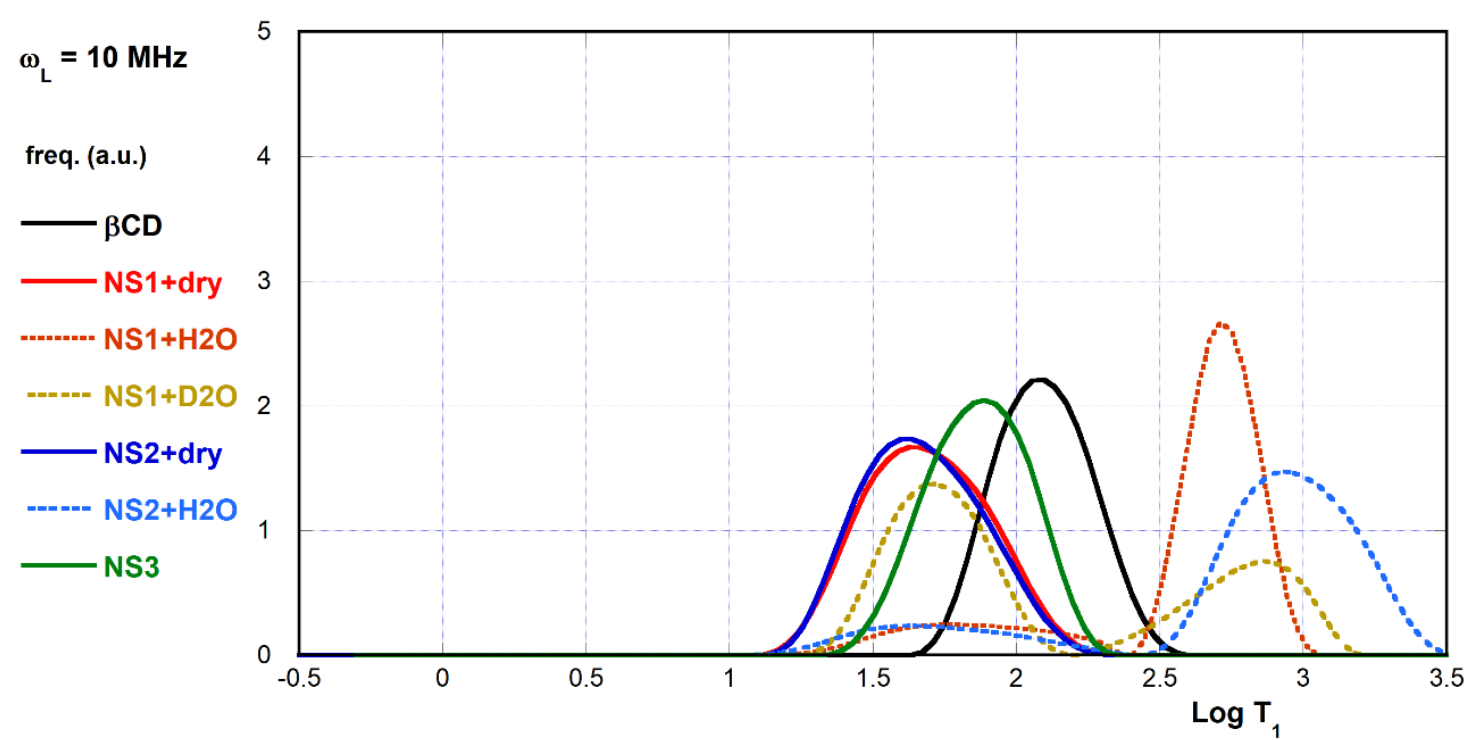


Figure S3. $T_{1}$ distribution curves at $3 \mathrm{MHz}$.

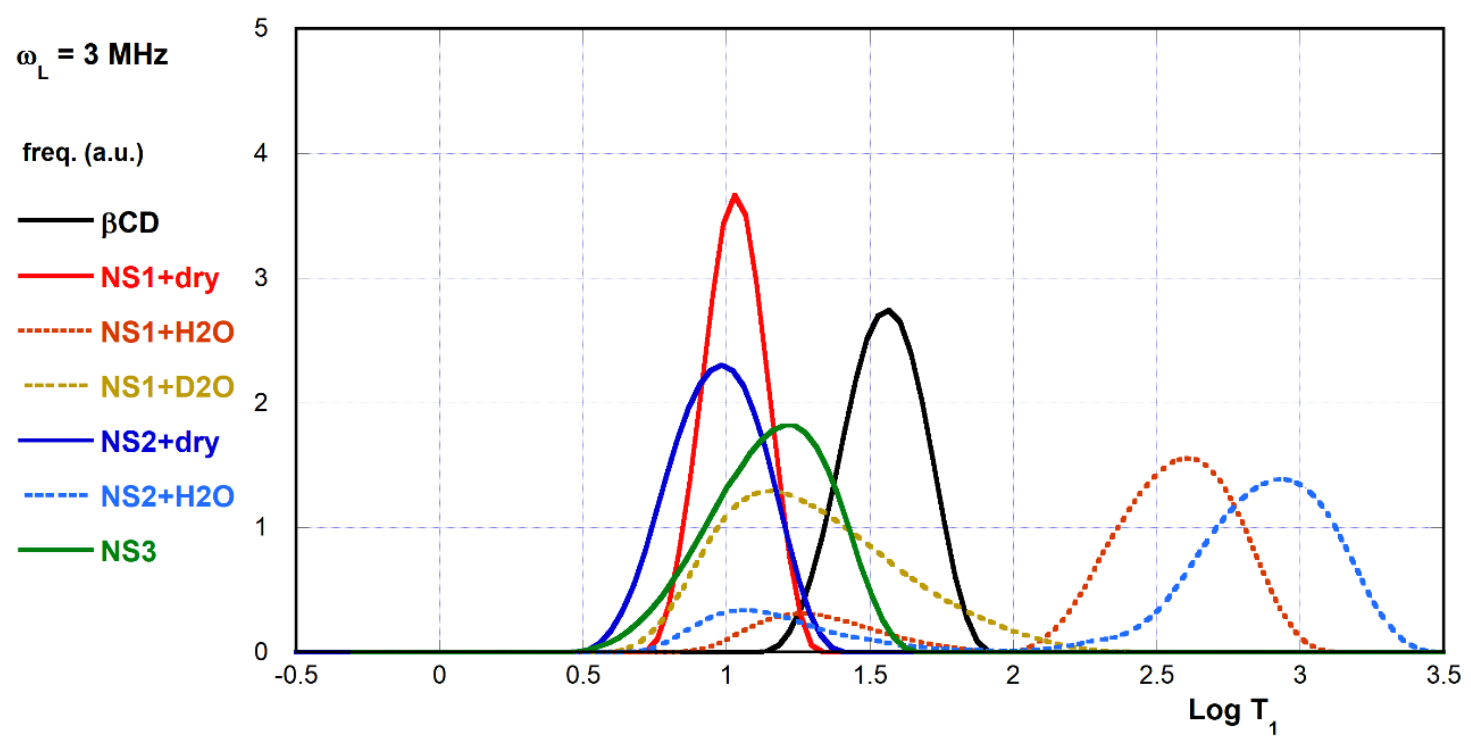

Figure S4. $T_{1}$ distribution curves at $1 \mathrm{MHz}$.

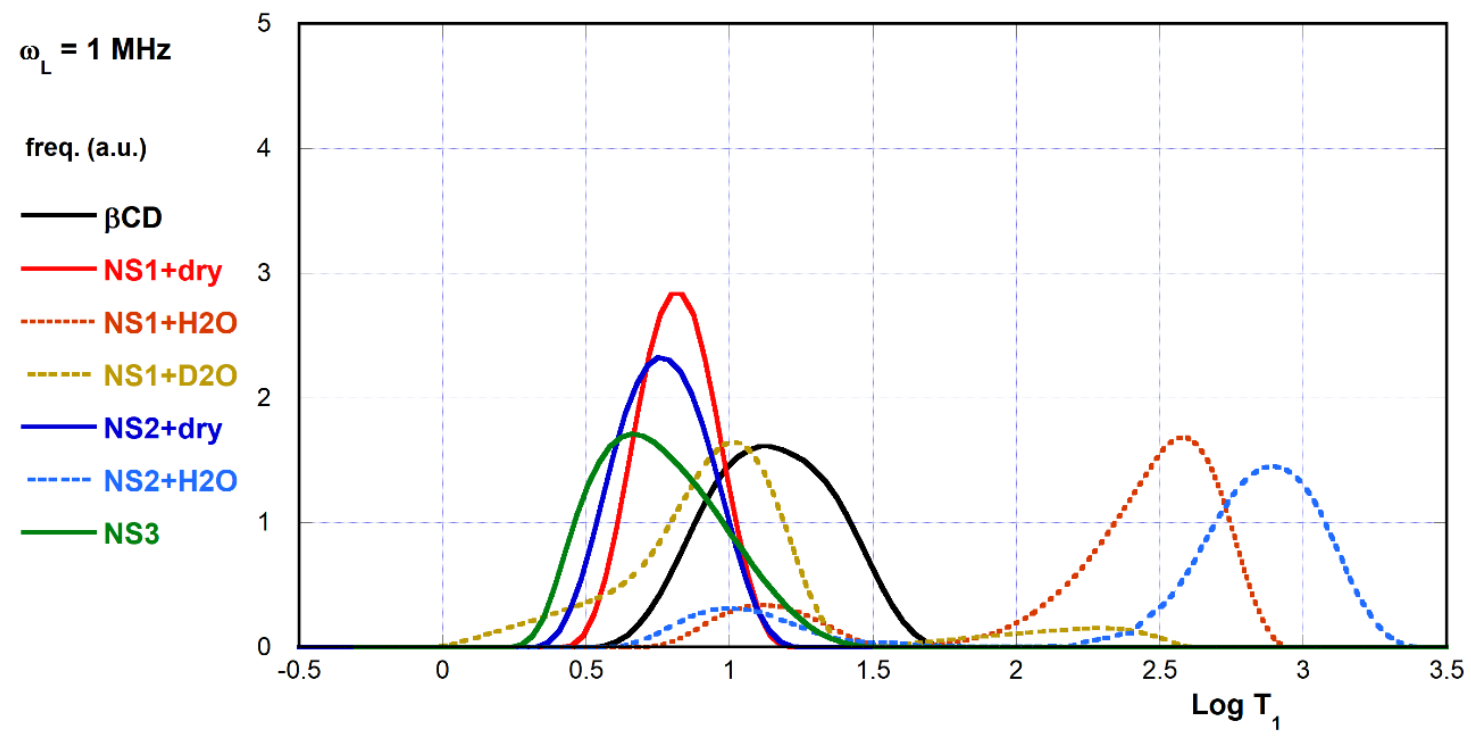


Figure S5. $T_{1}$ distribution curves at $0.3 \mathrm{MHz}$.

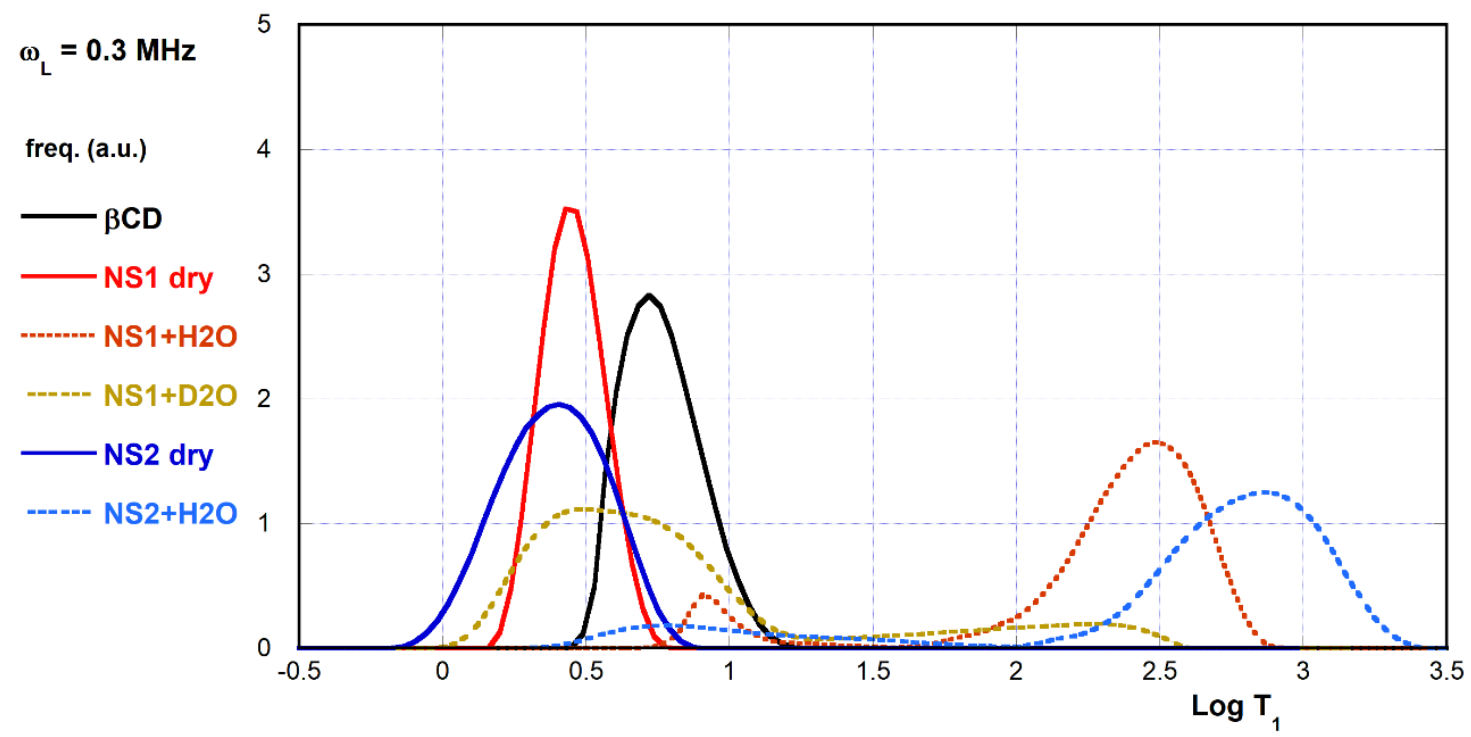


v) Table S3. Log-normal regression parameters for normalized Inverse-Laplace (UPEN) $T_{1}$ distribution curves.

$$
P(T)=\sum_{i}\left\{\frac{1}{\sqrt{2 \pi}} \cdot\left(\frac{p_{i}}{\sigma_{i}}\right) \cdot \exp \left[-\frac{1}{2}\left(\frac{\ln T-\ln T_{i}}{\sigma_{i}}\right)^{2}\right] \cdot \operatorname{erf}\left(a_{i} \cdot \frac{\ln T-\ln T_{i}}{\sigma_{i}}\right)\right\}
$$

\begin{tabular}{|c|c|c|c|c|c|}
\hline sample & $\omega_{L}(\mathrm{MHz})$ & $p_{\mathrm{i}}$ & $\sigma_{\mathrm{i}}$ & $\ln T_{\mathrm{i}}$ & $a_{\mathrm{i}}$ \\
\hline \multirow[t]{5}{*}{$\beta C D$} & 35 & 1 & $0.239 \pm 0.009$ & $2.359 \pm 0.012$ & $0.74 \pm 0.11$ \\
\hline & 10 & 1 & $0.209 \pm 0.007$ & $1.985 \pm 0.011$ & $0.62 \pm 0.09$ \\
\hline & 3 & 1 & $0.177 \pm 0.004$ & $1.650 \pm 0.006$ & $-0.72 \pm 0.06$ \\
\hline & 1 & 1 & $0.25 \pm 0.04$ & $1.09 \pm 0.14$ & $0.24 \pm 0.56$ \\
\hline & 0.3 & 1 & $0.225 \pm 0.003$ & $0.601 \pm 0.002$ & $1.96 \pm 0.09$ \\
\hline \multirow{5}{*}{ NS1 dry } & 35 & 1 & $0.141 \pm 0.001$ & $2.151 \pm 0.001$ & 0 \\
\hline & 10 & 1 & $0.296 \pm 0.010$ & $1.511 \pm 0.013$ & $0.81 \pm 0.10$ \\
\hline & 3 & 1 & $0.113 \pm 0.006$ & $1.06 \pm 0.02$ & $-0.27 \pm 0.17$ \\
\hline & 1 & 1 & $0.139 \pm 0.001$ & $0.816 \pm 0.001$ & 0 \\
\hline & 0.3 & 1 & $0.112 \pm 0.001$ & $0.450 \pm 0.001$ & 0 \\
\hline \multirow[t]{10}{*}{$\mathrm{NS} 1+\mathrm{H}_{2} \mathrm{O}$} & 35 & $0.804 \pm 0.005$ & $0.086 \pm 0.003$ & $2.924 \pm 0.005$ & $-0.51 \pm 0.08$ \\
\hline & & $0.196 \pm 0.003$ & $0.315 \pm 0.015$ & $2.501 \pm 0.008$ & $-2.6 \pm 0.4$ \\
\hline & 10 & $0.818 \pm 0.012$ & $0.121 \pm 0.001$ & $2.721 \pm 0.001$ & 0 \\
\hline & & $0.143 \pm 0.011$ & $0.27 \pm 0.02$ & $1.817 \pm 0.017$ & 0 \\
\hline & 3 & $0.841 \pm 0.009$ & $0.276 \pm 0.008$ & $2.743 \pm 0.008$ & $-0.90 \pm 0.08$ \\
\hline & & $0.159 \pm 0.010$ & $0.29 \pm 0.03$ & $1.11 \pm 0.03$ & $1.3 \pm 0.4$ \\
\hline & 1 & $0.849 \pm 0.013$ & $0.343 \pm 0.001$ & $2.745 \pm 0.001$ & $-2.48 \pm 0.04$ \\
\hline & & $0.151 \pm 0.013$ & $0.19 \pm 0.02$ & $1.04 \pm 0.04$ & $0.5 \pm 0.3$ \\
\hline & 0.3 & $0.912 \pm 0.014$ & $0.322 \pm 0.004$ & $2.659 \pm 0.003$ & $-1.8 \pm 0.5$ \\
\hline & & $0.088 \pm 0.011$ & $0.315 \pm 0.010$ & $0.850 \pm 0.006$ & $2.16 \pm 0.07$ \\
\hline \multirow[t]{10}{*}{$\mathrm{NS} 1+\mathrm{D}_{2} \mathrm{O}$} & 35 & 1 & $0.207 \pm 0.002$ & $2.098 \pm 0.001$ & $1.61 \pm 0.05$ \\
\hline & 10 & $0.372 \pm 0.007$ & $0.318 \pm 0.001$ & $3.018 \pm 0.005$ & $-2.06 \pm 0.18$ \\
\hline & & $0.628 \pm 0.008$ & $0.178 \pm 0.002$ & $1.717 \pm 0.002$ & 0 \\
\hline & 3 & 1 & $0.521 \pm 0.003$ & $0.917 \pm 0.002$ & $2.41 \pm 0.05$ \\
\hline & 1 & $0.087 \pm 0.011$ & $0.45 \pm 0.06$ & $2.47 \pm 0.03$ & $-3.5 \pm 1.8$ \\
\hline & & $0.295 \pm 0.015$ & $0.152 \pm 0.005$ & $1.125 \pm 0.006$ & 0 \\
\hline & & $0.618 \pm 0.014$ & $0.235 \pm 0.008$ & $0.816 \pm 0.016$ & 0 \\
\hline & 0.3 & $0.208 \pm 0.003$ & $0.90 \pm 0.05$ & $2.470 \pm 0.005$ & $-7.0 \pm 0.7$ \\
\hline & & $0.270 \pm 0.014$ & $0.187 \pm 0.016$ & $0.795 \pm 0.018$ & 0 \\
\hline & & $0.532 \pm 0.013$ & $0.32 \pm 0.07$ & $0.256 \pm 0.005$ & $1.62 \pm 0.44$ \\
\hline \multirow[t]{5}{*}{ NS2 dry } & 35 & 1 & $0.238 \pm 0.010$ & $2.039 \pm 0.013$ & $0.74 \pm 0.11$ \\
\hline & 10 & 1 & $0.318 \pm 0.007$ & $1.460 \pm 0.006$ & $1.16 \pm 0.08$ \\
\hline & 3 & 1 & $0.205 \pm 0.007$ & $1.081 \pm 0.010$ & $-0.66 \pm 0.08$ \\
\hline & 1 & 1 & $0.169 \pm 0.001$ & $0.768 \pm 0.001$ & 0 \\
\hline & 0.3 & 1 & $0.200 \pm 0.002$ & $0.391 \pm 0.002$ & 0 \\
\hline \multirow[t]{12}{*}{$\mathrm{NS} 2+\mathrm{H}_{2} \mathrm{O}$} & 35 & $0.812 \pm 0.008$ & $0.206 \pm 0.003$ & $3.267 \pm 0.005$ & $-1.519 \pm 0.02$ \\
\hline & & $0.046 \pm 0.014$ & $0.11 \pm 0.03$ & $2.41 \pm 0.05$ & 0 \\
\hline & & $0.142 \pm 0.012$ & $0.18 \pm 0.03$ & $2.10 \pm 0.04$ & 0 \\
\hline & 10 & $0.829 \pm 0.009$ & $0.273 \pm 0.012$ & $2.816 \pm 0.016$ & $0.76 \pm 0.12$ \\
\hline & & $0.052 \pm 0.013$ & $0.16 \pm 0.06$ & $2.00 \pm 0.09$ & 0 \\
\hline & & $0.127 \pm 0.014$ & $0.21 \pm 0.05$ & $1.60 \pm 0.07$ & 0 \\
\hline & 3 & $0.818 \pm 0.012$ & $0.33 \pm 0.06$ & $3.110 \pm 0.005$ & $-1.21 \pm 0.07$ \\
\hline & & $0.192 \pm 0.009$ & $0.37 \pm 0.02$ & $0.884 \pm 0.012$ & $2.32 \pm 0.45$ \\
\hline & 1 & $0.832 \pm 0.014$ & $0.294 \pm 0.005$ & $3.059 \pm 0.004$ & $-1.19 \pm 0.06$ \\
\hline & & $0.168 \pm 0.012$ & $0.30 \pm 0.02$ & $0.839 \pm 0.014$ & $1.47 \pm 0.28$ \\
\hline & 0.3 & $0.859 \pm 0.009$ & $0.373 \pm 0.008$ & $3.055 \pm 0.007$ & $-1.27 \pm 0.08$ \\
\hline & & $0.141 \pm 0.007$ & $0.53 \pm 0.08$ & $0.55 \pm 0.03$ & $3.0 \pm 1.2$ \\
\hline \multirow[t]{4}{*}{ NS3 } & 35 & 1 & $0.125 \pm 0.004$ & $2.317 \pm 0.007$ & $0.48 \pm 0.08$ \\
\hline & 10 & 1 & $0.233 \pm 0.008$ & $1.997 \pm 0.011$ & $-0.70 \pm 0.09$ \\
\hline & 3 & 1 & $0.331 \pm 0.004$ & $1.384 \pm 0.003$ & $-1.57 \pm 0.06$ \\
\hline & 1 & 1 & $0.378 \pm 0.005$ & $0.484 \pm 0.003$ & $2.10 \pm 0.10$ \\
\hline
\end{tabular}


vi) Table S4. $f_{\mathrm{S}}$ vs $\omega_{\mathrm{L}}$ data for NS1 and NS2.

\begin{tabular}{|c|c|c|c|}
\hline \multicolumn{2}{|c|}{ NS1 } & \multicolumn{2}{|c|}{ NS2 } \\
\hline$\omega_{\mathrm{L}}(\mathrm{MHz})$ & $f_{\mathrm{s}}$ & $\omega_{L}(\mathrm{MHz})$ & $f_{\mathrm{s}}$ \\
\hline 0.1 & $0.097 \pm 0.012$ & 0.1 & $0.035 \pm 0.003$ \\
\hline 0.3 & $0.086 \pm 0.011$ & 0.3 & $0.035 \pm 0.005$ \\
\hline 0.7 & $0.075 \pm 0.006$ & 0.7 & $0.039 \pm 0.003$ \\
\hline 1.0 & $0.068 \pm 0.006$ & 1.0 & $0.032 \pm 0.003$ \\
\hline 2.0 & $0.069 \pm 0.005$ & 1.3 & $0.032 \pm 0.002$ \\
\hline 2.1 & $0.068 \pm 0.005$ & 1.5 & $0.031 \pm 0.002$ \\
\hline 2.2 & $0.070 \pm 0.005$ & 1.7 & $0.032 \pm 0.002$ \\
\hline 2.3 & $0.069 \pm 0.005$ & 1.85 & $0.033 \pm 0.002$ \\
\hline 2.4 & $0.067 \pm 0.005$ & 2.0 & $0.034 \pm 0.002$ \\
\hline 2.45 & $0.071 \pm 0.004$ & 2.1 & $0.032 \pm 0.002$ \\
\hline 2.5 & $0.071 \pm 0.005$ & 2.3 & $0.032 \pm 0.002$ \\
\hline 2.6 & $0.064 \pm 0.005$ & 2.35 & $0.029 \pm 0.002$ \\
\hline 2.7 & $0.070 \pm 0.005$ & 2.4 & $0.030 \pm 0.002$ \\
\hline 2.8 & $0.072 \pm 0.005$ & 2.45 & $0.030 \pm 0.002$ \\
\hline 2.9 & $0.063 \pm 0.005$ & 2.5 & $0.030 \pm 0.002$ \\
\hline 3.0 & $0.066 \pm 0.005$ & 2.55 & $0.030 \pm 0.002$ \\
\hline 3.05 & $0.063 \pm 0.005$ & 2.6 & $0.030 \pm 0.002$ \\
\hline 3.1 & $0.063 \pm 0.005$ & 2.7 & $0.031 \pm 0.002$ \\
\hline 3.15 & $0.071 \pm 0.005$ & 2.8 & $0.032 \pm 0.002$ \\
\hline 3.2 & $0.070 \pm 0.005$ & 2.9 & $0.031 \pm 0.002$ \\
\hline 3.3 & $0.067 \pm 0.005$ & 2.95 & $0.031 \pm 0.002$ \\
\hline 3.4 & $0.070 \pm 0.005$ & 3.0 & $0.030 \pm 0.002$ \\
\hline 3.5 & $0.074 \pm 0.004$ & 3.1 & $0.030 \pm 0.002$ \\
\hline 4.0 & $0.081 \pm 0.004$ & 3.15 & $0.031 \pm 0.002$ \\
\hline 5.0 & $0.088 \pm 0.006$ & 3.2 & $0.032 \pm 0.002$ \\
\hline 6.0 & $0.090 \pm 0.006$ & 3.3 & $0.033 \pm 0.002$ \\
\hline 7.0 & $0.096 \pm 0.005$ & 3.4 & $0.034 \pm 0.002$ \\
\hline 8.0 & $0.095 \pm 0.005$ & 3.5 & $0.033 \pm 0.002$ \\
\hline 9.0 & $0.103 \pm 0.007$ & 3.7 & $0.035 \pm 0.002$ \\
\hline 10.0 & $0.105 \pm 0.005$ & 4.0 & $0.037 \pm 0.003$ \\
\hline 15.0 & $0.116 \pm 0.004$ & 5.0 & $0.040 \pm 0.002$ \\
\hline 20.0 & $0.129 \pm 0.003$ & 6.0 & $0.041 \pm 0.002$ \\
\hline 25.0 & $0.138 \pm 0.004$ & 7.0 & $0.040 \pm 0.002$ \\
\hline 30.0 & $0.149 \pm 0.004$ & 8.0 & $0.043 \pm 0.002$ \\
\hline \multirow[t]{12}{*}{35.0} & $0.153 \pm 0.003$ & 9.0 & $0.045 \pm 0.002$ \\
\hline & & 10.0 & $0.047 \pm 0.002$ \\
\hline & & 11.0 & $0.049 \pm 0.002$ \\
\hline & & 12.0 & $0.052 \pm 0.002$ \\
\hline & & 13.0 & $0.052 \pm 0.002$ \\
\hline & & 14.0 & $0.054 \pm 0.002$ \\
\hline & & 15.0 & $0.056 \pm 0.002$ \\
\hline & & 17.0 & $0.058 \pm 0.002$ \\
\hline & & 20.0 & $0.060 \pm 0.002$ \\
\hline & & 25.0 & $0.065 \pm 0.005$ \\
\hline & & 30.0 & $0.064 \pm 0.006$ \\
\hline & & 35.0 & $0.080 \pm 0.005$ \\
\hline
\end{tabular}

